EPJ Web of Conferences 18, 03002 (2011)

DOI: $10.1051 /$ epjconf/20111803002

(C) Owned by the authors, published by EDP Sciences, 2011

\title{
Gas-surface interactions and heterogeneous chemistry on interstellar grains analogues
}

\author{
J.H. Fillion ${ }^{1,2}$, F. Dulieu ${ }^{3}$, C. Romanzin ${ }^{1,2}$ and S. Cazaux ${ }^{4}$ \\ 1 UPMC Univ. Paris 06, CNRS, UMR 7092, LPMAA, 75005 Paris, France \\ 2 CNRS- UMR7092, LPMAA, 75005 Paris, France \\ ${ }^{3}$ LAMAp/LERMA, UMR 8112 du CNRS, Observatoire de Paris Université de Cergy Pontoise, \\ 5 mail Gay-Lussac, 95000 Cergy Pontoise Cedex, France \\ 4 Kapteyn Inst., 9700 AV Groningen, The Netherlands
}

\begin{abstract}
Detailed laboratory studies and progress in surface science technique, have allowed in recent years the first experimental confirmation of surface reaction schemes, as introduced by Tielens, Hagen and Charnley $[1,2]$. In this paper, we review few heterogeneous processes which give routes to form elementary molecules considered as precursors for explaining the variety and richness of molecular species in the interstellar medium. Adsorption, diffusion and reaction processes are discussed. With emphasis on the experimental approaches, but also supported by theoretical developments, progresses in the understanding of the "catalytic role" of a dust grain surface in various physical conditions are described. Recent advances made on few important species $\left(\mathrm{H}_{2}, \mathrm{H}_{2} \mathrm{O}, \mathrm{CH}_{3} \mathrm{OH}\right)$ are used to illustrate basic properties and raise open questions.
\end{abstract}

\section{INTRODUCTION}

Astrochemistry is fundamental to understand the formation of molecules in many locations in the universe, and especially in star forming regions where a wealth of molecular species is present. More than 150 molecules have been observed in interstellar and circumstellar environments, from the simplest and most abundant molecule of the universe $\mathrm{H}_{2}$, to traces of organic species with sizes up to 15 atoms. For more than 30 years, astronomers and chemists have investigated the gas-phase formation and destruction channels of various molecules from atomic matter. The understanding of the chemical evolution of the interstellar medium (ISM) has been a great stimulus to develop intense research programs around ion-molecule and neutral-neutral reactions. Theoretical models including a huge network of gas phase reactions are now able to explain most of the molecules detected in the gas phase $[3,4]$. It is now well recognized that gas-grain interactions are playing additional important role, in many regions of the interstellar space, enabling another - but still largely unknown - chemistry to occur through heterogeneous interaction between the gas and the surface of grains. This lack of knowledge about gas-grain interactions appears as a limitation for the interpretation of observations, especially in dense star forming regions, where the gas-dust interaction dominates any purely gas-phase process. Heterogeneous chemistry gives routes to form elementary molecules that are precursors for explaining the variety and richness of molecular species in the ISM. Important new efforts have been made to understand the interaction of atoms and molecules with surfaces of astrophysical interest, in physical conditions that are extreme and difficult to reproduce in the laboratory (low pressure, low temperature, intense radiation fields and fast protons and electrons). In this paper, we discuss some progress done in this area with emphasis made on experimental approaches.

This is an Open Access article distributed under the terms of the Creative Commons Attribution-Noncommercial License 3.0, which permits unrestricted use, distribution, and reproduction in any noncommercial medium, provided the original work is properly cited. 


\section{EPJ Web of Conferences}

Table 1. Elemental abundances with respect to total hydrogen.

\begin{tabular}{cccc}
\hline Element & Cosmic $^{(1)}$ & Diffuse Gas $^{(2)}$ & Dense Gas $^{(3)}$ \\
\hline $\mathrm{C}$ & $2.45(-04)$ & $1.32(-04)$ & $7.3(-05)$ \\
$\mathrm{O}$ & $4.6(-04)$ & $3.2(-04)$ & $1.76(-04)$ \\
$\mathrm{N}$ & $6.0(-05)$ & $7.5(-05)$ & $2.14(-05)$ \\
$\mathrm{S}$ & $2.6(-05)$ & $1.86(-05)$ & $8.0(-08)$ \\
$\mathrm{Si}$ & $1.38(-05)$ & $2.9(-05)$ & $8.0(-08)$ \\
\hline
\end{tabular}

(1) Photosperic abundances [8]; (2) $\zeta$ Persei [6]; (3) Ref [9].

\subsection{Gas and dust in the interstellar medium}

As described in other chapters of this book, the interstellar medium contains both gas phase atoms and molecules as well as particles (dust or "grains") that are synthesized in stars. Dust grains contribute for approximately $1 \%$ of the mass of the material in interstellar cloud, with a number density of 1.3 $10^{-12}$ that of the gas phase. In diffuse interstellar clouds, the gas densities are $10^{2}-10^{4} \mathrm{~cm}^{-3}$, gas phase temperatures range from 50 to $100 \mathrm{~K}$ and grain temperature is about $20 \mathrm{~K}$ [5]. The ultraviolet and visible radiation from stars can penetrate the cloud, reducing the concentrations of molecules by photodissociation and photoionisation. The gas is thus mainly in atomic from, except for hydrogen which is also in molecular form as it becomes self shielded. Small abundances of diatomic $\left(\mathrm{CH}, \mathrm{CH}^{+}\right.$, $\left.\mathrm{OH}, \mathrm{NH}, \mathrm{C}_{2}, \mathrm{CO}\right)$, triatomic species $\left(\mathrm{H}_{3}^{+}, \mathrm{C}_{3}\right)$ and polyatomics molecules [6] have been detected in absorption using background stars. Dusts consist of amorphous silicates and various forms of carbonaceous material [5]. Grains are distributed in several domains depending on their size. There are "standard grains" (diameter a $>0,025 \mu \mathrm{m}$ ) which contribute for the extinction, polarization and scattering at visible wavelengths, "very small grains" (a<0,0025 $\mu \mathrm{m})$ which contributes to the extinction in the vacuum ultraviolet, and the molecular domain (few $\AA$ in size) that include PAHlike species, having graphitic properties. The non-spherical shape of some grains may cause selective extinction of starlight observed with polarization. Large interstellar grains might have properties that are similar to interplanetary dust particles, in term of shape, size, morphology and composition, suggesting porous, fluffy and even fractal morphologies [7]. Typical abundances with respect to total hydrogen density $\mathrm{n}_{0}$ are given in table 1 . The depletion of the elements, as compared with stellar atmospheres ("cosmic" abundances) suggests that these elements are likely to form the dust grain material.

In dense clouds, gas densities $\left(10^{4}-10^{6} \mathrm{~cm}^{-3}\right)$ and dust concentrations are higher, giving a high extinction with very little UV and visible light penetrating. In the gas phase, $\mathrm{H}_{2}$ is the most abundant by far, with $\mathrm{CO}$ in second place (four orders of magnitude lower in concentration), and a large number of molecular species, that can be identified via rotational spectroscopy in the millimeter wave region. In dense regions, the gas and dust temperature can be very low (typically $10 \mathrm{~K}$ ). The grains are thus covered by icy mantles containing mainly water. The inventory of ices towards embedded protostars and various other objects [10-13] have shown that the mantles contains significant amount of simple saturated molecules: $\mathrm{CO}_{2}, \mathrm{CO}, \mathrm{CH}_{3} \mathrm{OH} \mathrm{H} \mathrm{H}_{2} \mathrm{CO}, \mathrm{CH}_{4}, \mathrm{NH}_{3}, \mathrm{HCOOH}$, and other traces of nitrogen and sulfur species $\mathrm{OCN}^{-}$, OCS. During their lifetime, the grains can be processed by UV photons, energetic ion bombardment or low energy-electrons (few hundred meV). Molecules that have been synthesized can be ejected from the grain and participate to the gas phase chemistry. Alternatively, molecules or radicals can be trapped in the ice mantle and contribute to chemistry within the bulk of the ice mantle. In the vicinity of protostars, the ice mantles evaporate, providing a wealth of new molecular species released in the gas phase. Indeed, high abundances of organic species are observed in "hot cores" around high mass protostars [14], or "hot corinos" around low mass protostars [15-17]. The energetic processes (thermal, photolysis or ion-irradiation) occurring in the bulk of ice mantles have been thoroughly investigated 
Table 2. Gas phase densities $\left(\mathrm{cm}^{-3}\right)$ in dense cloud [27].

\begin{tabular}{cccccc}
\hline total density $\mathrm{n}_{0}\left(\mathrm{~cm}^{-3}\right)$ & $\mathrm{H}$ & $\mathrm{O}$ & $\mathrm{N}$ & $\mathrm{C}$ & $\mathrm{CO}$ \\
\hline $10^{4}$ & 1 & 2.0 & $8.5(-02)$ & $4.2(-02)$ & 1.35 \\
$10^{5}$ & 1 & $2.0(01)$ & $8.5(-01)$ & $4.2(-01)$ & $1.35(01)$ \\
\hline
\end{tabular}

since the 1980s and led to the identification of many products and yields [18-23] that are not discussed here.

The present paper focuses on non-energetic processes that are occurring, either on the external top-layers of ices, or directly onto the surface of bare grains. At first sight, low temperatures and low densities found in the interstellar medium are not favorable to chemistry, since one can generally believe that thermal activation is necessary. Heterogeneous chemistry is however required to explain the chemical origin of some molecular species, among which molecular hydrogen has attracted the attention of astrochemists for a long time. Molecular hydrogen is however far to be a single case, since many other species that can be detected in solid ices, except $\mathrm{CO}$, are probably synthesized through gas-surface interactions and catalytic processes.

There are different roles that the interaction with grain can play. At first, the accretion of the gas on dust depletes molecular species from the gas at high density, which can impact dramatically the gas phase chemistry. This "passive" role occurs for instance in the freeze out of CO which is a main destroyer of species like $\mathrm{N}_{2} \mathrm{H}^{+}, \mathrm{H}_{2} \mathrm{D}^{+}$or $\mathrm{D}_{3}^{+}$[24]. Dissociative electron recombination of $\mathrm{H}_{2} \mathrm{D}^{+}$ produces in particular D-atoms which results in high degree of deuterium fractionation in dense regions $([D] /[H] \geq 0.3)[25]$. With the low densities present in the ISM, the collision rates can be very low in the gas phase (less than once a day), which limits the reaction rate thus governed by the accretion rate. With the presence of grains, the atoms and molecules can however encounter by accreting atoms and molecules on their cold surface, which acts as a third body for catalyzing chemical reactions. Tables 1 and 2 show that atoms $(\mathrm{H}, \mathrm{D}, \mathrm{C}, \mathrm{O}, \mathrm{N}$ and $\mathrm{S})$ are primordial reactants due to their abundances and high mobility. Indeed, light species (especially $\mathrm{H}$ ) are expected to be extremely mobile once adsorbed on the surface of the grains, which increases their probability to react. Reactions with radicals and atoms are in addition expected to have mostly low activation energy at low surface temperature. In diffuse regions, the role of $\mathrm{H}$ atoms is expected to be prominent and has been predicted to lead saturated hydrogenated species such as $\mathrm{H}_{2} \mathrm{O}, \mathrm{CH}_{4}, \mathrm{NH}_{3}$. In denser regions, $\mathrm{H}$ is in molecular form $\left(\mathrm{H} / \mathrm{H}_{2} \sim 10^{-3}\right)$ [26] and the density of remaining $\mathrm{H}$ atoms is determined by the balance between the destruction of $\mathrm{H}_{2}$ by cosmic rays and the formation of $\mathrm{H}_{2}$ on surfaces. The $\mathrm{H}$-atoms density is of the order of $1 \mathrm{~cm}^{-3}$, whatever the total density of the cloud. Unlike $\mathrm{H}$, the abundance of other atoms like $\mathrm{O}\left(\mathrm{O} / \mathrm{H}_{2} \sim 10^{-4}\right)$ increases with density and can become more abundant than atomic hydrogen (table 2), leading to other species such as $\mathrm{N}_{2}, \mathrm{O}_{2}$ or $\mathrm{CO}_{2}$.

Although gas-grain chemical schemes have been introduced in the 80 's by Tielens, Hagen and Charnley [1, 2], experimental evidences and associated theoretical developments for the heterogeneous chemistry on astrophysical relevant surfaces have emerged only in recent years. This paper propose a review of some of these studies, which involve hydrogen and oxygen atoms interacting either on bare grain surface (relevant for diffuse regions) or on icy mantles (relevant for denser regions).

\subsection{Gas and dust in the laboratory}

Historically, laboratory investigations of astrophysical ices were performed in high vacuum, giving information about the spectroscopy through the techniques of cold matrices [28] or processes occurring in the bulk of solid ices [29]. This has led to the identification of major components of astrophysical ices and shown that photochemical and thermal processes could lead to more complex organic species 
EPJ Web of Conferences

Table 3. Comparison of experimental conditions with those found in the ISM.

\begin{tabular}{lll}
\hline Parameter & Laboratory & ISM (dense) \\
\hline density in the gas & $10^{6} \mathrm{~cm}^{-3}$ & $10^{4} \mathrm{~cm}^{-3}$ \\
surface temperature & $10-300 \mathrm{~K}$ & $10-200 \mathrm{~K}$ \\
atomic gas temperature & $40-1500 \mathrm{~K}$ & $10-100 \mathrm{~K}$ \\
atomic flux & $10^{12}-10^{15} \mathrm{~cm}^{-2} \mathrm{~s}^{-1}$ & $10^{9} \mathrm{~cm}^{-2} \mathrm{~s}^{-1}$ \\
$\mathrm{H} / \mathrm{H}_{2}$ & $0.6-0.8$ & 0.5 \\
$\mathrm{O} / \mathrm{O}_{2}$ & 0.3 & $10^{2}$ \\
\hline
\end{tabular}

[30]. Stimulated by astronomers, gas-phase and surface scientists have developed experiments in ultrahigh vacuum conditions, suited for the study of processes occurring on macroscopic samples. Although valuable analogues of carbon grains [31-33] or silicates [26] can now be obtained in the laboratory, only very few experimental studies of the chemical reactivity of such samples have been undertaken up to now, except in the case of $\mathrm{H}_{2}$ formation on amorphous carbon [34] and amorphous/crystalline silicates [35], or for the hydrogenation of carbon nanograins [36, 37]. The complexity and the variety of materials that can be produced by different techniques (vapor condensation, arc-discharge, laser vaporization, thermal heating or burning, plasma deposition...) have been evidenced [26]. Most of the chemical studies concerning wet-surface samples have been focused (both experimentally and theoretically) on graphite (or graphene) surfaces, as they are considered as an ideal prototype for carbon bearing material and PAH-like species. The surface on which most of the studies have been performed at low temperatures is amorphous solid water (ASW), that can be grown by vapor condensation on carbon or silicate base or directly on an inert cold metallic substrate. Given the absence of gravity and extreme conditions found in space, these films might not be able to reproduce the diversity of structures present in space [38]. Nevertheless, ASW is believed to be highly relevant to simulate the icy waterdominated mantle of interstellar grain and has in addition the great interest to present various stable states at low temperatures. By changing the experimental deposition method and the temperature at which slow deposition rates are made (typically $<0.5 \mathrm{ML} / \mathrm{s}$, where $1 \mathrm{ML}=10^{15}$ molecules cm $^{-2}$ ), it is possible to form porous ASW, compact ASW or crystalline ice deposits [39]. One can control the effective surface area interacting with the gas, that can reach few thousands $\mathrm{m}^{2} / \mathrm{g}$ in the case of highly porous films [40, 41]. Experiments suggest that energetic ion bombardments and UV irradiation should lead to a compaction of the ice films under interstellar conditions [42-44]. However, the question of the morphology (and possible porosity) of the nascent water ice formed by heterogeneous chemistry has only very recently been explored, as shown in the next sections. Due to this high sensitivity to the deposition method, various preparation protocols of the ice films used in different laboratories gives results that are obviously difficult to compare to each other. Consequently, a "standard laboratory ice film" (density, porosity, composition) is highly desirable in order to facilitate the interpretations.

Table 3 shows that many of the parameters involved in the ISM can be reproduced in the laboratory. In an ultra-vacuum chamber, the residual gas consists of $\mathrm{H}_{2}, \mathrm{CO}$ and traces of $\mathrm{CO}_{2}$. Although one could claim that these conditions mimic that of interstellar environments, residual gas can in general be neglected on the time scale of a typical experiment. For example, with a typical base pressure of $10^{-10}$ mbar, a cold sample can be covered by residual gas in 3 hours. When the sample is irradiated by atomic beams, the residual molecular gas present in the beam can however cover the surface in much less time and long exposures should therefore in principle be avoided. In such conditions, one can mimic atom exposures equivalent to that found over $10^{3}-10^{6}$ years in molecular clouds.

In 1995, G. Vidali and V. Pironnello started a series of elegant experimental investigations with the aim to address the problem of the formation of molecular hydrogen $[35,45,46]$. In these experiments, 
the formation processes in conditions as close as possible to that encountered in the ISM are simulated on surfaces of astrophysical relevance, although such surfaces are of course only partly known. The sample surface, either olivine, amorphous carbon or solid water ice, is irradiated by two neutral atomic beams (one H, one D) at fixed temperature and for a given period of time. The sample is then heated to measure the rate of HD desorption as a function of temperature, providing the so called "Thermally Programmed Desorption" experiment (TPD) curves. This remains the only experimental determination of the formation efficiency of $\mathrm{H}_{2}$ on olivine [35] and amorphous carbon [47]. In this type of experiment, atoms stick, migrate, encounter, recombine and are released in the gas phase. Thus, important efforts in modeling such experiments had to be made to disentangle the different steps of the catalyzing process. These models are based on strong hypothesis concerning the mechanisms and on physical parameters that are mostly unknown. In the following section, we review some processes that have been recently investigated and which provide quantitative information on the sticking probabilities, mobility (including barriers for diffusion), reaction efficiencies (including barriers for reactions), and energy partitioning, with the aim to elucidate the key reactions occurring under interstellar conditions.

\section{PROCESSES}

The collision of an atom or molecule with a surface can lead to direct (or non direct) scattering back to the gas phase. Alternatively, the atom (molecule) can stick to the surface, by multiple collisions and kinetic energy transfer to the surface. Its residence time on the surface can however be very short, depending on the surface temperature and interactions forces. It can migrate on the surface before reaching a binding site through weak van der Waals interaction (physisorption) or stronger interaction such as hydrogen bonding or chemical binding with an atom of the surface (chemisorption). In some cases, the incident atom, radical or ion can collide directly on another species generating a new species without adsorbing, following the so-called Eley-Rideal mechanism (ER). In other cases, the two reactants can follow the so-called Langmuir-Hinshelwood mechanism (LH) in a "bimolecular" reaction. In this latter case, reactants have to meet indirectly on the surface, by thermal diffusion, quantum tunneling, or due to incident kinetic energy. Modeling heterogeneous chemistry requires a quantitative determination of these elementary steps.

\subsection{Adsorption}

In the ISM, dust is supposed to represent $1 \%$ of the matter. Its size distribution classically follows the law proposed by Mathis et al. [48]. The total grain surface area $S_{g}$ available for the gas-surface interaction is proportional to the density $\mathrm{n}_{0}$ of the cloud. The accretion flux $(\mathrm{F})$ on grains of a species $\mathrm{X}$ is $\mathrm{F}_{\mathrm{X}}=\mathrm{n}_{\mathrm{X}} \mathrm{v}_{\mathrm{X}} \mathrm{S}_{\mathrm{g}}$ where $\mathrm{n}_{\mathrm{X}}$ is the density, $\mathrm{v}_{\mathrm{X}}$ the speed of the species $\mathrm{X}$. Given that the density $\mathrm{n}_{\mathrm{X}}$ of a species $X$ is related to $n_{0}$, the accretion flux is proportional to $n_{D}^{2}$. Hence, the density of the cloud regulates the accretion flux and is a dominant factor for gas-grain interactions.

The second main factor is the grain surface temperature. Indeed, the sticking coefficient $\mathrm{S}$, defined as the probability for a gas phase species to thermally equilibrate with the grain, exhibits only a slight dependence to the grain temperature. The key factor is therefore the desorption rate as compared with the accretion rate. When the desorption rate exceeds that of the accretion rate, the species are maintained in the gas phase. In the opposite case, species accrete onto the grain.

Considering the case of physisorbed species, adsorption/desorption is linked to the polarisability of each species. Highly polar species such as $\mathrm{H}_{2} \mathrm{O}, \mathrm{CO}_{2}$ and $\mathrm{CH}_{3} \mathrm{OH}$ can accrete at "high" temperature (80-100 K), whereas species such as $\mathrm{CO}$ and $\mathrm{N}_{2}$, accrete around $20 \mathrm{~K}$ and even at lower temperatures in the case of $\mathrm{H}$ and $\mathrm{H}_{2}$. Yet as previously pointed, it also slightly depends on other physical parameters such as $\mathrm{n}_{\mathrm{X}}$, and the gas temperature. The desorption rate of relevant molecules has been qualitatively studied by Collings et al. [49] and has been the subject of numerous studies in recent years, as reviewed recently by Burke and Brown [50]. 


\section{EPJ Web of Conferences}

The third factor that influences the accretion rate is the gas temperature. Firstly, the speed $v_{x}$ of the species varies as $\sqrt{T}$ and changes the incoming flux by the same factor. Secondly, the gas temperature also affects the sticking coefficient. The sticking process is mostly governed by the ability of a species to decrease its initial kinetic energy to be finally trapped on the surface. Therefore, a high adsorption energy and good momentum transfer increase the sticking efficiency. The sticking of the gas species onto a cold surface can be divided in two categories depending on their mass. The light particules such as $\mathrm{H}$, $\mathrm{H}_{2}, \mathrm{D}, \mathrm{D}_{2}, \mathrm{H}$ and $\mathrm{He}$ on one side, and the other heavier species on the other side. The light particules have a small mass $\left(\mathrm{m}<4\right.$ a.u.) compared to the atoms composing the grains $\left(\mathrm{C}, \mathrm{O}, \mathrm{Si}\right.$ or $\mathrm{H}_{2} \mathrm{O}$ if capped), and low binding energy, linked to their weak polarisability. By contrast, the sticking of heavy elements benefits from of a good momentum transfer with the surface due to better mass matching between the two colliding partners and due to a larger adsorption energy. Thus, sticking coefficients of heavy species are usually assumed to be close to unity. Experimental studies confirm that sticking efficiencies of $\mathrm{O}_{2}, \mathrm{~N}_{2}, \mathrm{CO}, \mathrm{CH}_{4}$, and $\mathrm{H}_{2} \mathrm{O}$ exceed $90 \%$ [41, 51, 52]. On the contrary, the sticking of light molecules and atoms has raised numerous questions. Especially, the sticking of $\mathrm{H}$-atoms on graphite is of high importance for the formation of $\mathrm{H}_{2}$ (see section 2.3.2 below). Besides, the sticking of $\mathrm{H}$ on water ice is crucial for explaining the formation of number of hydrogenated species at the surface of ice mantles in dense clouds [53].

The sticking of H-atoms chemisorbed onto graphite (0001) can be as large as 0,4 at $150 \mathrm{~K}$ [54], but is smaller at low temperature and show strong isotopic effect as the lattice dynamics and tunnelling effect are taken into account [55]. On ASW, the H-atom sticking probability decreases rapidly with incident energy and surface temperature, as shown by several calculations [56-58]. In astrophysics models, the sticking is generally assumed to vary with the kinetic temperature $\mathrm{T}$ as $\sqrt{T / 10}$, which cancels the $\mathrm{T}$ dependence of the accretion rate and gives apparent constant formation rate, as observed for $\mathrm{H}_{2}$. The sticking efficiency of $\mathrm{H}$ on water ice has been predicted to be similar on crystalline and amorphous ice [53]. Recently, Matar et al. [59] have performed the first experimental study of hydrogen and deuterium molecules as a function of incident beam temperature. These authors have proposed a statistical model that highlights the mass-dependence relation between the sticking behaviour of $\mathrm{H}_{2}$ and $\mathrm{D}_{2}$ with temperature, together with the sticking of $\mathrm{H}$ and $\mathrm{D}$ atoms. The expression $S(T)=S_{0}\left(1+\beta T / T_{0}\right) /\left(1+T / T_{0}\right)^{\beta}$ for the sticking of $\mathrm{H}$ on amorphous ice has been obtained, with the parameters $\mathrm{S}_{0}=1, \beta=2.5$ and $\mathrm{T}_{0}=52 \mathrm{~K}$, and can be implemented directly into simulations of the ice mantle formation. High values of the sticking efficiency on ASW ( $>90 \%$ ) have been found for these light species at low temperature [59].

\subsection{Diffusion}

In gas phase, the interaction between two species occurs during a collision that can be reactive or nonreactive (the two bodies separate definitively). If two species are stuck together on a cold surface, there can be a large number of collisions ("attempts for reaction") before they separate. Therefore a low kinetic rate for a reaction can be compensated by a long presence of both of the reactants. The total chemical production on dust grains is deeply ruled by the ability of the reactant to move on the surface of the grain and to find each other. The diffusion, mobility or even miscibility of the species is a key point to understand the chemistry in solid phase. We will treat here the case of the physisorption which should be the dominant process at low temperature.

\subsubsection{Case of mobility on a frozen template}

It is usually assumed that an adsorbed species can move from one adsorption site to another by a simple hop. The grain surface is supposed to be unaltered by adsorption. The probability to move from one site to another is usually described by the crossing of a barrier. In most cases the crossing rate $\mathrm{G}$ is correctly described by an Arrhenius law $\mathrm{G} \sim \mathrm{e}^{-\mathrm{E}_{\mathrm{d}} / \mathrm{KT}}$ where $\mathrm{E}_{\mathrm{d}}$ is the diffusion energy barrier. The desorption is 
usually also described by an Arrhenius law involving in this case the adsorption energy $\mathrm{E}_{\mathrm{a}}$. Very little is known about the relation between the hopping barrier and the desorption energy. It is typically assumed in astrophysical models that $\frac{1}{3} \mathrm{E}_{\mathrm{a}}<\mathrm{E}_{\mathrm{d}}<\frac{2}{3} \mathrm{E}_{\mathrm{a}}$, and one commonly estimates $\mathrm{E}_{\mathrm{d}} \sim 0.5 \mathrm{E}_{\mathrm{a}}$ when no other value is available $[60,61]$. The probability to hop is always higher than the probability to desorb. The ratio between mobility and desorption is a way to estimate the number of hops prior to desorption. This number can be written: $\mathrm{e}^{-\mathrm{E}_{\mathrm{d}} / \mathrm{kT}} / \mathrm{e}^{-\mathrm{E}_{\mathrm{a}} / \mathrm{kT}} \sim \mathrm{e}^{+\mathrm{E}_{\mathrm{a}} / 2 \mathrm{kT}}$. This expression gives large numbers in most cases, meaning basically that a species physisorbed on a surface will scan the surface area entirely before its desorption. In some extreme cases, the diffusion barrier is very weak $(<4 \mathrm{meV})$ in comparison with the adsorption energy $(\sim 40 \mathrm{meV})$, and the adsorbed species can be considered as freely mobile even at $10 \mathrm{~K}$. This situation is found for example in the case of $\mathrm{H}$-atoms physisorbed on a coronene, or on hexagonal ice [62]. This is mostly due to the very regular geometry of the surface. On the contrary, in the case of very amorphous surface, it is demonstrated that the disordered topology can trap locally even $\mathrm{H}$, the most mobile reactive atom, as show for example on ASW [63]. But if this localised sites can trap reactants, they cannot prevent other atoms to move and to reach them, this is why, in the case of amorphous solids, the mobility is clearly linked to the coverage on the surface. As soon as the tiny fraction of trapping sites $(<1 \%)$ is populated, a mean diffusion barrier can be estimated [64]. This diffusion barrier is therefore typical of physisorption on a more regular substrate.

Finally, regardless of the diffusion barrier, quantum tunnelling which doesn't require to overcome the barrier, can always happen. Basically, it is a general rule that the kinetics of a barrier-crossing transition is dominated by tunneling below the crossover temperature: $T_{c}=\hbar \Omega / 2 \pi k_{B}$ where $\Omega$ is the magnitude of the imaginary frequency of the unstable normal mode at the saddle point of the potential energy surface ("the barrier") [65]. At temperature $\mathrm{T}_{\mathrm{c}}$, the full quantum rate is typically a few times larger (2-3) than the classical (thermal hopping) rate. That means that there is a temperature regime around $\mathrm{T}_{\mathrm{c}}$ where tunneling and thermal hopping rates are of similar magnitude, i.e., where the system goes from an Arrhenius behaviour at high T to only tunneling at low T. See Ref [66] for an illustration the $\mathrm{CH}_{4}+\mathrm{H}$ system and Ref [67] for a discussion of $\mathrm{H}$ and D diffusion on grains.

\subsubsection{Case of mixing and segregation of the substrate}

In the context of dense clouds, the surface of the grains is made of adsorbed molecules such as $\mathrm{H}_{2} \mathrm{O}$, $\mathrm{CO}_{2}, \mathrm{CO}, \mathrm{H}_{2}$ and other molecules mentioned in the introduction. $\mathrm{H}_{2} \mathrm{O}$ and $\mathrm{CO}_{2}$ can be considered to form the ice bulk at $10 \mathrm{~K}$, and the question is more centred on the role of $\mathrm{H}_{2}$ that can also be present on the surface. It has been shown that $\mathrm{H}_{2}$ plays a role in the energetic balance by de-exciting newly formed $\mathrm{H}_{2}$ molecule on nonporous ASW [68], but also by increasing the sticking coefficient of the atoms [69]. Between 15 and $25 \mathrm{~K}$, the mobility of $\mathrm{O}_{2}$ and $\mathrm{CO}$ has been demonstrated [64], and it can for example change the production rate of water [70]. At higher temperatures (80-120 K), $\mathrm{H}_{2} \mathrm{O}$ and $\mathrm{CO}_{2}$ segregate [71] due to diffusion within the bulk in competition with desorption. As it will be discussed in section 2.3, the diffusion of molecules (especially water) is also directly related to proton exchanges mechanisms and isotopic fractionation [72] which places the mobility of species in the heart of poorly known catalytic processes. Segregation and transformation mechanisms are at early stage and should be further explored in the coming years.

\subsection{Reaction}

\subsubsection{Formation of molecular hydrogen}

Molecular hydrogen is the most abundant molecule in our Universe. Observations of many astrophysical environments revealed that this molecule could form and survive in extreme conditions such as strong UV/X-rays fields, cold and warm gas and dust, or post-shocks regions. It has been recognized very early that $\mathrm{H}_{2}$ forms preferably on dust grains in the ISM and that this process is more efficient than gas phase 
routes by many orders of magnitude [73]. However, the real processes that allow this molecule to form on dust for a wide range of physical conditions have been the dedication of many studies in the last decades.

The formation of $\mathrm{H}_{2}$ at low dust temperatures involves physisorbed $\mathrm{H}$ atoms. Experiments on silicates and carbonaceous dust grain analogues at low surface temperatures [34, 35] showed that the formation of $\mathrm{H}_{2}$ involving 2 physisorbed atoms (Langmuir-Hinshelwood mechanism) is a very efficient process. This has been confirmed by rate equations and Monte Carlo simulations [67, 74, 75] that showed that this efficiency could reach $100 \%$ until $\sim 25 \mathrm{~K}$. Also, three-dimensional wave packet calculations showed very high efficiencies depending on collision energies of the adsorbed $\mathrm{H}$ atoms, which would produce $\mathrm{H}_{2}$ molecules with a considerable amount of vibrational energy and with a ortho/para ratio corresponding to the high temperature limit $[76,77]$. Some of this vibrational energy can be converted into rotational energy by successive collisions between the newly formed $\mathrm{H}_{2}$ and the pore walls of the carbonaceous surface [78]. The formation of $\mathrm{H}_{2}$ in excited rovibrational states has been confirmed experimentally on graphite between 15 and $50 \mathrm{~K}$ [79-82] but also on non-porous amorphous ice at $10 \mathrm{~K}$ [83]. However, $\mathrm{H}_{2}$ can also form at low temperatures from the reaction of a gas phase $\mathrm{H}$ that hits a physisorbed atom (Eley-Rideal mechanism). Indeed, in interstellar cloud conditions, the Eley-Rideal mechanism involves mostly physisorbed $\mathrm{H}$ in comparison with chemisorbed $\mathrm{H}$ [84].

By considering the roughness of the surface of dust grains, which would allow $\mathrm{H}$ atoms to be bound to the surface following a distribution in energies, $\mathrm{H}_{2}$ can form with physisorbed $\mathrm{H}$ atoms until higher dust temperatures $(40 \mathrm{~K})$ [75]. However, as this process could explain the formation of $\mathrm{H}_{2}$ in diffuse clouds and in some PDRs where dust is cold, observations of warm PDRs, shocks in young stellar objects and supernovae remnants revealed that molecular hydrogen exists and is formed in regions with much warmer grains. Observations of several PDRs, for which a $\mathrm{H}_{2}$ formation efficiency could be derived [85], have shown that $\mathrm{H}_{2}$ is able to form on dust until almost $100 \mathrm{~K}$. For warm dust temperatures ( $>20-30 \mathrm{~K}$ ), physisorbed atoms evaporate from the grain surface, and other processes are necessary to explain the formation of $\mathrm{H}_{2}$ at high temperatures. These processes usually involve chemisorbed $\mathrm{H}$ for the formation of $\mathrm{H}_{2}$ and are detailed for different surfaces in the next paragraphs.

Many studies concentrate on graphite surfaces for their relative simplicity to treat experimentally as well as theoretically. By using thermal desorption spectroscopy (TDS), it has been shown that $\mathrm{H}_{2}$ could form on graphite surfaces at temperatures of few hundreds of Kelvins [86]. This study proved that $\mathrm{H}_{2}$ could form at high dust temperatures, involving chemisorbed atoms, and allowed to address the importance of this mechanism in the formation of $\mathrm{H}_{2}$ [67]. At high grain temperature, the formation of $\mathrm{H}_{2}$ is insured by $\mathrm{H}$ atoms coming from the gas phase that hit chemisorbed $\mathrm{H}$ atoms on the surface (EleyRideal mechanism). Density Functional theory calculations (DFT) showed that $\mathrm{H}$ atom chemisorption onto graphite occurs only atop a $\mathrm{C}$ atom that puckers out of the basal plane to allow the $\mathrm{CH}$ bond to form [87-90]. This puckering is associated with an activation barrier of $0.2 \mathrm{eV}$ for gas phase $\mathrm{H}$ atoms to enter chemisorbed sites. This barrier highly restricts the access of gaseous $\mathrm{H}$ to chemisorbed sites, making the formation of $\mathrm{H}_{2}$ on graphite much less efficient.

Nevertheless, recent studies that focused on the adsorption of $2 \mathrm{H}$ atoms on graphite surfaces, showed that once one $\mathrm{H}$ atom is present on the surface, a second $\mathrm{H}$ atom (with a spin opposite to the first) could become chemisorbed without a barrier in the so-called "para" site, where the two H atoms are adsorbed on opposite sides of the same carbon ring. By contrast, this effect doesn't hold in the socalled "ortho" sites, where the two atoms are adsorbed on two nearest carbons [84, 91-93]. Scanning tunneling microscopy revealed the existence of distinct hydrogen dimers states on graphite basal planes, as shown in figure 1 (left). Direct recombination from dimer states into molecular hydrogen takes place with a barrier of $1.4 \mathrm{eV}$ for para dimers and $1.6 \mathrm{eV}$ for ortho dimer states (figure 1, right), in good agreement with the barriers derived from the TPD spectra measured by Zecho et al. 2002. Also, recent DFT calculations from Ferro et al. [94], investigate the magnetism and the stability of the different dimers on the graphene. The results confirm that the para and ortho dimers are the most stable dimers structures, as revealed by STM images (figure 1, left). 

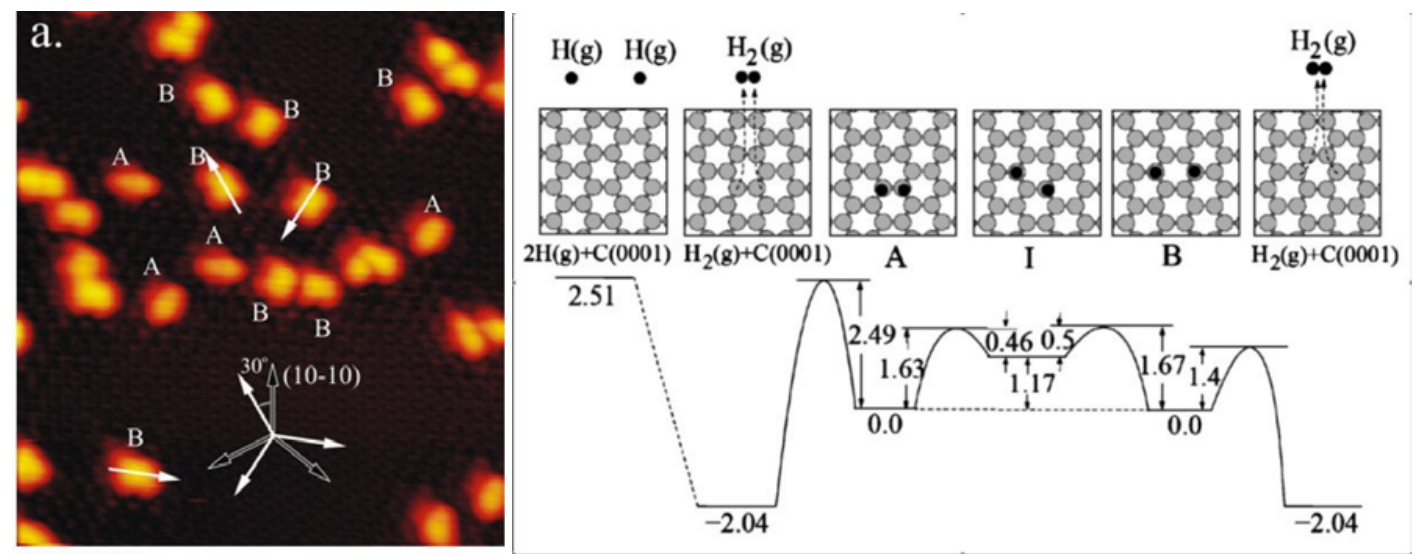

Figure 1. STM image of dimer structures. Energy barriers for atomic hydrogen diffusion and recombination from dimer A and B states on the graphite surface [93].

Once a para dimer is formed on the surface, a third $\mathrm{H}$ atom coming from the gas phase can interact and react with one of the 2 adsorbed atoms, and form $\mathrm{H}_{2}$ without a barrier [95]. This mechanism provides a barrier-less route for the formation of $\mathrm{H}_{2}$ on graphitic surfaces and increases the $\mathrm{H}_{2}$ formation efficiency by 1 order of magnitude [96].

The formation of dimers seems to be the results of fast diffusion of physisorbed $\mathrm{H}$ atoms that become chemisorbed in a dimer configuration, or to dimer mediated Eley-Rideal mechanism [97]. STM images reveal that at high surface coverage, other structures such as star/triangles are observed that exhibit identical orientations of the surface [98]. Ferro et al. [99], by using DFT calculations, assigned the star structures to hydrogenated hexamers on the surface of graphite. These hexamers are some highly symmetric structures with an axis of order six. They are made of three para dimers whose stability is now well established. They exhibit an enhanced stability as compare to three isolated para dimers [94, 99]. As a consequence, the star structures are the most stable $\mathrm{H}$ clusters on the surface of graphite. The fact that $\mathrm{H}$ atoms can group in clusters with high binding energies provides new mechanisms to insure the formation of $\mathrm{H}_{2}$ at very high surface temperatures.

While large PAHs are usually treated as graphite surfaces, the contribution of the aliphatic $\mathrm{CH}_{2,3}$ groups for the formation of $\mathrm{H}_{2}$ are usually neglected. Recent studies [37] show that an $\mathrm{H}$ atom coming from the gas phase that encounters an aliphatic group to form $\mathrm{H}_{2}$ is a barrier-less reaction and that this mechanism can contribute for a large fraction of the $\mathrm{H}_{2}$ formation rate in diffuse clouds and PDRs.

\subsubsection{Hydrogenation reactions with oxygen bearing molecules}

Hydrogenation reactions are expected to be among the most important surface reactions on interstellar ices in cold environments, because of the relative high abundance of hydrogen in the dense interstellar medium (see table 2) and because of its high mobility at low temperature, as discussed above. There are number of oxygen bearing molecules, such as $\mathrm{CO}, \mathrm{CO}_{2}, \mathrm{H}_{2} \mathrm{CO}, \mathrm{CH}_{3} \mathrm{OH}, \mathrm{HCOOH}$ and $\mathrm{CH}_{3} \mathrm{CHO}$, that are present in interstellar ice mantles and may react with $\mathrm{H}$ atoms. These species are believed to be very important precursors to more complex organic molecules that can form in the ice at higher temperatures or by energetic processing due to UV photons and cosmic rays bombardment.

$\mathrm{CO}$ being a major component of ice mantle, its hydrogenation has been investigated in priority by exposing to partial $\mathrm{H}$-atom beams various ices such as pure solid $\mathrm{CO}, \mathrm{CO}-\mathrm{H}_{2} \mathrm{O}$ or $\mathrm{H}_{2} \mathrm{CO}-\mathrm{H}_{2} \mathrm{O}$ mixed ices below $20 \mathrm{~K}$, [100-110]. These experiments clearly demonstrate that the formation of formaldehyde $\mathrm{H}_{2} \mathrm{CO}\left(\mathrm{D}_{2} \mathrm{CO}\right)$ and methanol $\mathrm{CH}_{3} \mathrm{OH}\left(\mathrm{CD}_{3} \mathrm{OD}\right)$ could proceed by successive addition of hydrogen 


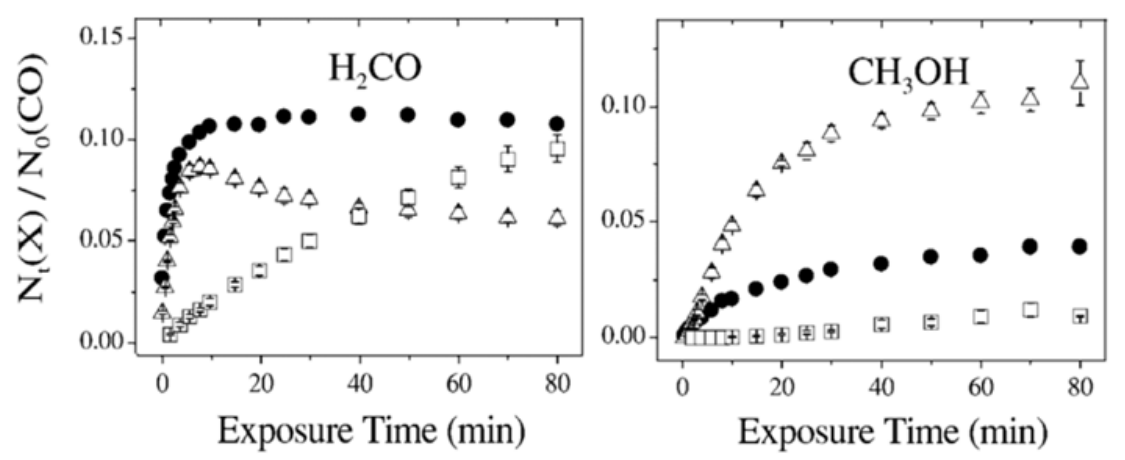

Figure 2. Variation as a function of $\mathrm{H}$-atoms exposure of $\mathrm{H}_{2} \mathrm{CO}$ (left) and $\mathrm{CH}_{3} \mathrm{OH}$ (rigth) column densities normalized with respect to the initial $\mathrm{CO}$ exposure. The sample is a $\mathrm{H}_{2} \mathrm{O}-\mathrm{CO}$ ice mixture $\left(\mathrm{H}_{2} \mathrm{O} / \mathrm{CO} \sim 4\right)$ at $10 \mathrm{~K}$ (filled circles), $15 \mathrm{~K}$ (open triangles) and $20 \mathrm{~K}$ (open squares). Figure adapted from Ref [111].

(deuterium).

$$
\begin{aligned}
\mathrm{CO} \stackrel{(1)}{\longrightarrow} \mathrm{HCO} \stackrel{(2)}{\longrightarrow} \mathrm{H}_{2} \mathrm{CO} \stackrel{(3)}{\longrightarrow} \mathrm{CH}_{3} \mathrm{O} \text { or } \mathrm{CH}_{2} \mathrm{OH} \\
\mathrm{CH}_{3} \mathrm{O} \stackrel{\text { (4) }}{\longrightarrow} \mathrm{CH}_{3} \mathrm{OH}
\end{aligned}
$$

At low H-atoms flux $\left(\sim 10^{12} \mathrm{~cm}^{-2} \mathrm{~s}^{-1}\right)$, only $\mathrm{H}_{2} \mathrm{CO}$ could be detected in the experiments, whereas at high incident atomic flux $\left(10^{14}-10^{15} \mathrm{~cm}^{-2} \mathrm{~s}^{-1}\right)$, complete hydrogenation leading to $\mathrm{CH}_{3} \mathrm{OH}$ was observed [102]. None of these products could be observed when solid $\mathrm{CO}$ is exposed to molecular hydrogen, which is consistent with the fact that the reactions $\mathrm{X}+\mathrm{H}_{2} \rightarrow \mathrm{HX}+\mathrm{H}$ are endothermic or only slightly exothermic in the gas phase. Therefore, the unavoidable adsorption of $\mathrm{H}_{2}$ present in the atomic beam could be neglected on the chemical point of view. Another important experimental evidence is the non-observation of $\mathrm{HCO}$ and $\mathrm{CH}_{3} \mathrm{O}$ radicals, indicating that radical-radical reactions (steps 2 and 4 of the above sequence) proceeds much faster than other steps in the successive $\mathrm{H}$ addition. On the contrary, the hydrogenation of $\mathrm{CO}$ and $\mathrm{H}_{2} \mathrm{CO}$ (steps 1 and 3) are believed to proceed via tunneling, because these reactions have activation energies of few thousand $\mathrm{K}$ in the gas phase. For illustration, the abundances of $\mathrm{H}_{2} \mathrm{CO}$ and $\mathrm{CH}_{3} \mathrm{OH}$ that are formed from a CO- $\mathrm{H}_{2} \mathrm{O}$ ice mixture irradiated by $\mathrm{H}$-atoms are shown in figure 2 . The rise of $\mathrm{H}_{2} \mathrm{CO}$ is almost the same for surfaces at $10 \mathrm{~K}$ and $15 \mathrm{~K}$ (see first 10 minutes in figure 2), which is consistent with a reaction proceeding by tunneling effect. By contrast, the yields for $\mathrm{CH}_{3} \mathrm{OH}$ are clearly higher when the surface temperature is $20 \mathrm{~K}$, in agreement with a thermally activated reaction for the formation of $\mathrm{CH}_{3} \mathrm{OH}$ from $\mathrm{H}_{2} \mathrm{CO}$. Energy barriers for the $\mathrm{CO}+\mathrm{H}$ and $\mathrm{H}_{2} \mathrm{CO}+\mathrm{H}$ reactions have been obtained by fitting Monte Carlo simulations to experimental data [109].

Other experiments using simultaneous irradiation of $\mathrm{CO}$ and $\mathrm{H}$ do show intermediates reactants such as $\mathrm{HCO}[112,113]$ and $\mathrm{CH}_{3} \mathrm{O}$ radicals [113] on the contrary to the sequential irradiation experiments discussed just above. The column densities of these radicals seems to depend strongly on both temperature and $\mathrm{H}$ concentration, indicating that surface specific reactions (revealed through sequential deposition experiments) and bulk reactions (revealed via co-deposition experiments) proceed differently. In the first case, the $\mathrm{H}$ atoms penetrate hardly within the ice at very low temperature (typically $4 \mathrm{ML}$ at $12 \mathrm{~K}$ ) [109], even if better penetration could however explain the important yields observed for $\mathrm{CH}_{3} \mathrm{OH}$ at $15 \mathrm{~K}$ in figure 2.

Experiments conducted on $\mathrm{H}_{2} \mathrm{O}-\mathrm{CO}$ ice mixtures with different thicknesses do show higher yields in the case of thin samples $(<10 \mathrm{ML})$, for which the ratio of $\mathrm{CO}$ molecules present at the surface with respect to the total amount of $\mathrm{CO}$ is higher. The actual density of reactants present on the surface (or surface coverage) appears to be a key parameter, partly governed by the solid ice geometry and 


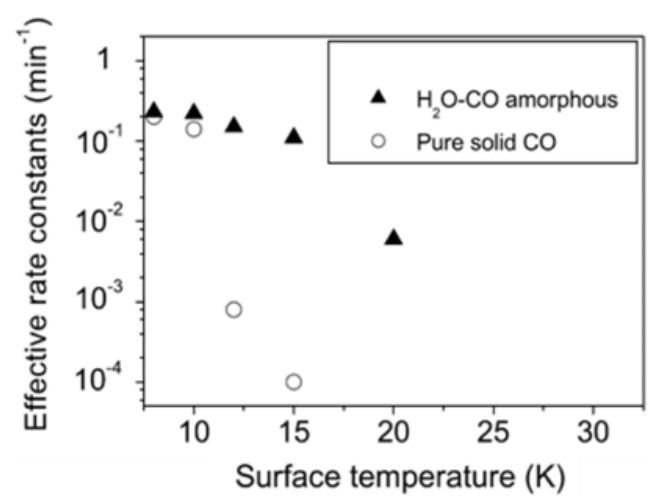

Figure 3. Effective rate constants for hydrogenation of CO from [110].

composition. In surface hydrogenation experiments, the formation of successive layers, where $\mathrm{H}_{2} \mathrm{CO}$ formed over the $\mathrm{CO}$ sample limits the interaction of $\mathrm{H}$ with $\mathrm{CO}$, and where $\mathrm{CH}_{3} \mathrm{OH}$ formed over $\mathrm{H}_{2} \mathrm{CO}$ prevents its interaction with gas phase hydrogen atoms. This effect might explain the saturation of $\mathrm{H}_{2} \mathrm{CO}$ to $\mathrm{CH}_{3} \mathrm{OH}$ formation at long exposure time in figure 2.

Rate constants can be derived from experiments. A correct treatment would require solving a set coupled kinetic rate equations associated to the different species. A simple approach can be used by considering the depletion of the parent molecule with time. When a species X reacts with an $\mathrm{H}$ (or D) atom to give $\mathrm{XH}$, its depletion is governed by:

$$
\frac{\mathrm{dN}}{\mathrm{dt}}=-\mathrm{k}_{\mathrm{H}} \mathrm{N}_{\mathrm{H}} \mathrm{N}_{\mathrm{X}},
$$

where $\mathrm{k}_{\mathrm{H}}$ is a standard reaction rate constant, $\mathrm{n}_{X}$ the surface density for species $\mathrm{X}$. One difficulty is to estimate the actual density $\mathrm{N}_{\mathrm{H}}$ of $\mathrm{H}$-atoms present at surface. Depending on the experimental conditions, more or less $\mathrm{H}$-atoms recombine to form $\mathrm{H}_{2}$ (up to 99,9\% in the case of Ref [111] for example). In most cases, one can consider a steady state regime, in which $\mathrm{N}_{\mathrm{H}}$ is constant. Consequently, one can fit the single exponential decay of the species $\mathrm{X}$ with time in order to obtain an effective rate constant $\mathrm{k}=\mathrm{k}_{\mathrm{H}} \mathrm{n}_{\mathrm{H}}$ as given in table 3 for the hydrogenation of various ices.

In a series of experimental investigations, the effects of various parameters such as the surface temperature, the surface composition, surface thickness and morphology [104-106, 108] and H-atom flux [109] were investigated. As shown in figure 3, the efficiencies for the hydrogenation are sensitive to ice temperature, composition and structure. At low temperature, the H-atoms reactivity is limited by slow diffusion. At higher temperatures, $\mathrm{H}$ desorption competes with hydrogenation. Several factors favoring longer residence time of hydrogen atoms tends to increase the temperature domain at which reaction can take place. $\mathrm{H}$ atoms are less tightly bound on pure $\mathrm{CO}$ ice than on $\mathrm{ASW}$ or $\mathrm{CO}-\mathrm{H}_{2} \mathrm{O}$ mixed ices leading to a rapid fall of effective constant with temperature (figures 2 and 3). The presence of other molecules, such as $\mathrm{H}_{2} \mathrm{CO}$ on ASW also enhances effective rates by increasing the adsorption energies of the H-atoms. Another factor is the roughness of the surface that favors higher binding energies due to multiple interactions between $\mathrm{H}$ and few surrounding water molecules. It should be pointed out that effective rates deduced from experiments also include the sticking efficiency of $\mathrm{H}$-atoms on the surface. Thus, non linear behavior of the effective rates can arise from the variation of the sticking probability with coverage (even due to non reactive species - such as $\mathrm{H}_{2}$ - present on the surface). In contrast with ASW substrates, effective rates constant for the $\mathrm{CO}$ hydrogenation are 5-10 times lower for polycrystalline ice, for the same amount of $\mathrm{CO}$ adsorbed on the surface. Nevertheless, when effective rate constants are scaled with respect to the surface area available on crystalline and porous ice respectively, equivalent rates are found, suggesting that the reactivity mostly depends on coverage, as 
EPJ Web of Conferences

Table 4. Effective rate constants for the hydrogenation of ices.

\begin{tabular}{cccc}
\hline Reaction & $\mathrm{T}_{\text {ice }}(\mathrm{K})$ & $\mathrm{k}\left(\mathrm{min}^{-1}\right)$ & Ref \\
\hline $\mathrm{CO}+\mathrm{H}$ & 10 & $1.4(-1)$ & {$[117]$} \\
$\mathrm{CO}+\mathrm{D}$ & 10 & $1.4(-2)$ & {$[117]$} \\
$\mathrm{H}_{2} \mathrm{CO}+\mathrm{H}$ & 10 & $1.4(-1)$ & {$[103]$} \\
$\mathrm{O}_{2}+\mathrm{H}$ & 10 & $1.2(+1)$ & {$[117]$} \\
$\mathrm{CH}_{3} \mathrm{CHO}+\mathrm{H}$ & 14.6 & $1.3(+2)$ & {$[115]$} \\
$\mathrm{CH}_{3} \mathrm{OH}+\mathrm{H}$ & 10 & $5.1(-1)$ & {$[118]$} \\
$\mathrm{HCOOH}$ & 12.5 & $<2.3(-17)$ & {$[115]$} \\
\hline
\end{tabular}

already stated above. Surprisingly, as compared with gas phase reactions, intrinsic rate constants appear to be not significantly affected by the presence of water, as shown by ab-initio calculations performed with up to four water molecules and for the hydrogenation of $\mathrm{CO}$ and $\mathrm{H}_{2} \mathrm{CO}$ [114].

Finally, few effective rates constants are given in table 3 for various ices. The hydrogenation of $\mathrm{CH}_{3} \mathrm{CHO}$ leads to the formation of $\mathrm{CH}_{4}, \mathrm{H}_{2} \mathrm{CO}, \mathrm{CH}_{3} \mathrm{OH}$ and in less extent to $\mathrm{C}_{2} \mathrm{H}_{5} \mathrm{OH}(<20 \%) . \mathrm{CO}_{2}$ and $\mathrm{HCOOH}$ do not react with $\mathrm{H}$ at a detectable level [115]. In some circumstances, absolute rate constants could be derived $[115,116])$ although this parameter is difficult to obtain from the experiments described in this section.

The very low rates observed for the $\mathrm{CO}+\mathrm{D}$ reaction has led to the conclusion that methanol containing D-atoms proceeds via a succession of tunneling $\mathrm{H}$-abstraction followed by $\mathrm{D}$ addition, rather than the successive D addition from $\mathrm{CO}[110,119,120]$. In addition, this process was shown to occur preferentially on methyl sites rather than on hydroxyl sites, as revealed by the non observation of $\mathrm{CH}_{3} \mathrm{OD}$ in the experiment [110]. This seems in line with observations of high abundance of deuterated molecules in low-mass protostars [121, 122], where under-abundance of $\mathrm{CH}_{3} \mathrm{OD}$ with respect to other methanol isotopomers has been revealed [123]. Complementary to the abstraction/addition process, H/D exchange in the hydroxyl group has been observed at higher temperature $(120-170 \mathrm{~K})$ in $\mathrm{H}_{2} \mathrm{O}-\mathrm{CH}_{3} \mathrm{OD}$ mixtures [124]. H/D isotopic exchanges were observed in other systems, such as between $\mathrm{H}_{2} \mathrm{O}$ and $\mathrm{D}_{2} \mathrm{O}$ on ice films at $90-140 \mathrm{~K}$. The exchanges are believed to be mediated by the motion of protonic defects $\left(\mathrm{H}_{3} \mathrm{O}^{+}\right)$, which occur preferentially at the surface layer of the ice [125]. It was also shown that $\mathrm{H} / \mathrm{D}$ exchanges could be greatly enhanced by the presence of excess proton concentration at surface, and by its propagation within the few top-layers of the ice facilitated by molecular re-orientations and diffusion of water above 125-130 K [126]. Considering now the extremely slow heating rates involved in the ISM, such events might in fact occur around $100 \mathrm{~K}$ in space [127], concomitantly to the phase transformation of ASW towards cubic crystalline ice and the desorption of (less volatile) ASW [128]. Such effect would strongly affect the diagnostic of solid state chemistry that could be inferred from molecular deuterium fractionation.

\subsubsection{Formation of water}

As recalled in the introduction, water is the main component of the icy mantles covering interstellar dust grains. The ice absorption features identified in various sources, such as embedded young stellar objects or quiescent dark clouds are consistent with amorphous water ice [13][129]. In most lines of sight, the water component appears to account for $\sim 60 \%$ of the ice [130]. Such abundances cannot be explained by direct condensation on the grains of water molecules formed through gas phase processes and water is thus believed to be mainly formed on dust grains by surface reactions [131-133].

Tielens and Hagen [1] have proposed a solid state reaction scheme where water molecules are formed by reactions involving $\mathrm{O}, \mathrm{O}_{2}$ and $\mathrm{O}_{3}$ as shown in table 4. Ozone is likely to be formed on 
Table 5. Possible formation routes for solid water.

\begin{tabular}{ccc}
\hline (i) Direct reaction & (ii) Hydrogenation of $\mathrm{O}_{2}$ & Hydrogenation of $\mathrm{O}_{3}$ \\
\hline $\mathrm{O}+\mathrm{H} \rightarrow \mathrm{OH}(1)$ & $\mathrm{O}_{2}+\mathrm{H} \rightarrow \mathrm{HO}_{2}(4)$ & $\mathrm{O}_{3}+\mathrm{H} \rightarrow \mathrm{OH}+\mathrm{O}_{2}(7)$ \\
$\mathrm{OH}+\mathrm{H} \rightarrow \mathrm{H}_{2} \mathrm{O}(2)$ & $\mathrm{HO}_{2}+\mathrm{HH}_{2} \mathrm{O}_{2}(5)$ & \\
$\mathrm{OH}+\mathrm{H}_{2} \rightarrow \mathrm{H}_{2} \mathrm{O}+\mathrm{H}(3)$ & $\mathrm{H}_{2} \mathrm{O}_{2}+\mathrm{HH}_{2} \mathrm{O}+\mathrm{OH}(6)$ & \\
\hline
\end{tabular}

the grain by reactions of $\mathrm{O}$ with $\mathrm{O}_{2}$ [1] or by energetic processes [134-137]. The $\mathrm{OH}$ radicals and the $\mathrm{O}_{2}$ molecule resulting from reaction (6) and/or (7) further lead to $\mathrm{H}_{2} \mathrm{O}$ via reactions (2) or (3) and (4) to (6) respectively. In this model, the $\mathrm{O}_{3}$ channel was found to be the most efficient whereas $\mathrm{O}_{2}$ would more play a catalytic role.

Theoretical investigations on the influence of the astrophysical environment have also been performed by using a Monte-Carlo approach [61]. The formation routes to water ice have been found to differ whether it occurs in dense or diffuse clouds. In dense clouds, water appears to form mainly through reactions (3) and (6) while, in diffuse clouds, the reaction between $\mathrm{H}$ and $\mathrm{OH}$, namely reaction (1), becomes predominant. The growth of the icy mantle was also found to be much less efficient under the conditions of diffuse interstellar clouds (submonolayer regime). In addition, Cuppen et al. [61] provides a first attempt to elucidate the structure of water ices built up from surface reactions. Actually little is known on this aspect. On a more general point of view, experimental data on these low-temperature solid state reactions are largely lacking although experimental evidence is needed in order to discriminate and evaluate the respective contribution of the different channels.

Despite some pioneering work on the hydrogenation of solid $\mathrm{O}_{2}$ and $\mathrm{O}_{3}$ performed in the late 50 's [138, 139], experiments dedicated to the study of heterogeneous reactions responsible for water formation are very recent. Successive hydrogenations following the first pathway (i) have been investigated by Biener et al. [140] at $85 \mathrm{~K}$ on $\mathrm{O}, \mathrm{OH}$ and $\mathrm{O}_{2}$-covered $\mathrm{Pt}(111)$ surfaces. Investigations at lower temperatures (10-12 K) were performed by Hiraoka et al. [100] using $\mathrm{O}$ atoms trapped in an $\mathrm{N}_{2} \mathrm{O}$ matrix and by Dulieu and co-workers [141] bombarding ASW with $\mathrm{H}$ and $\mathrm{O}$ atoms. These experiments were based on TPD techniques and concluded that O-atoms are efficiently converted into water. However, when $\mathrm{O}$ and $\mathrm{H}$ atoms directly interact, contribution of the channel (ii) must also be taken into account since $\mathrm{O}_{2}$ and, to a lower extent, $\mathrm{O}_{3}$ molecules are likely to be formed.

Experimental investigations on the hydrogenation of solid $\mathrm{O}_{2}$ (channel (ii)) are available from three independent studies either using direct absorption IR measurements (figure 4) and/or mass spectrometry but all confirming the subsequent formation of both hydrogen peroxide $\left(\mathrm{H}_{2} \mathrm{O}_{2}\right)$ and water at $10 \mathrm{~K}$ $[64,117]$ and up to $27 \mathrm{~K}$ [70]. Kinetic data have also been obtained, as shown in table 3 although different fitting models, respectively $1^{\text {st }}$ vs. $0^{\text {th }}$ order, were used to extract the data [70, 117]. The $\mathrm{O}_{2}$ hydrogenation experiments performed in Leiden [70] indeed further show that the formation yield of both $\mathrm{H}_{2} \mathrm{O}_{2}$ and $\mathrm{H}_{2} \mathrm{O}$ is temperature-dependent whereas initial reaction rates appear to be independent from the substrate temperature. This unexpected behaviour could be explained by considering the competition between the reaction of $\mathrm{H}$-atoms with $\mathrm{O}_{2}$ and the hydrogen diffusion into the ice, resulting in a $0^{\text {th }}$ order rate. In these experiments [70] no significant isotopic effect have been observed for the rate of reaction (6) on the contrary to what might be expected from the corresponding gas phase activation energy value $(\sim 2000 \mathrm{~K})$ and from previous results by Miyauchi et al. [117]. This might be due to the fact that, in addition to reactions (2) to (6), other reactions may be involved in the hydrogenation process and have to be taken into account to fit the data [70, 142]. Follow-up experiments should provide new insights concerning the reaction network [142]. Nevertheless, it is already clear from those experiments that reaction (4) has a negligible barrier and proceeds efficiently at low temperatures unlike what was previously assumed in astrochemical models. 

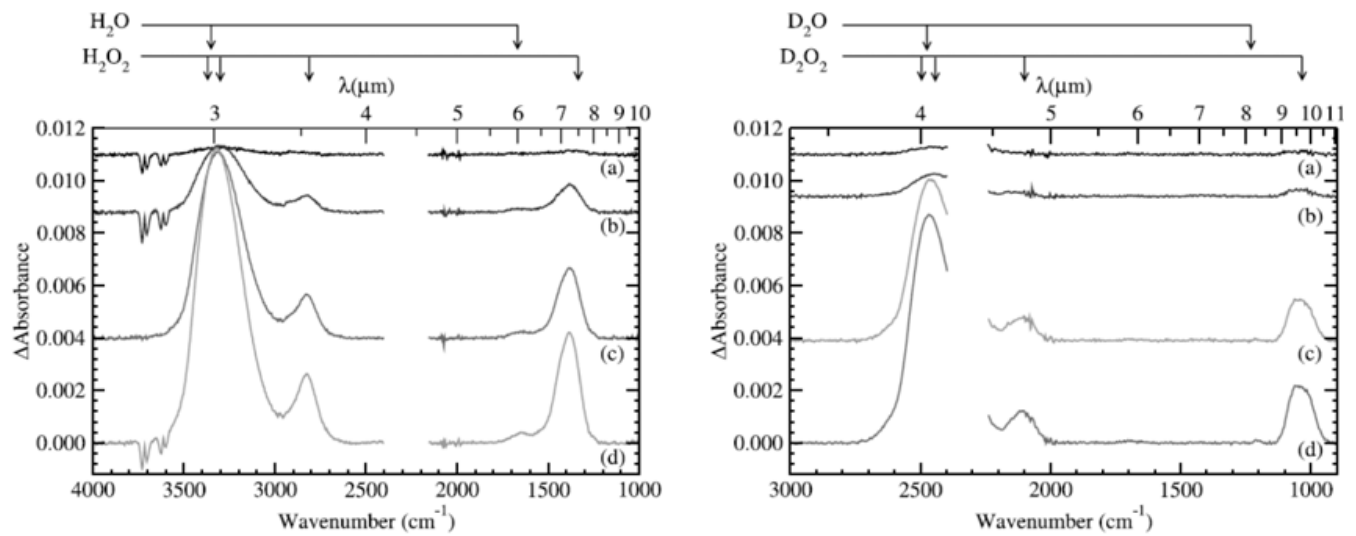

Figure 4. Reflexion Absorption InfraRed spectral changes of the $\mathrm{O}_{2}$ ice as a function of $\mathrm{H}$-atom (left) and D-atom (right) bombardment. Spectra at a H(D)-atom fluence of (a) $4 \times 10^{15}$, (b) $4 \times 10^{16}$, (c) $1 \times 10^{17}$, and (d) $2 \times 10^{17} \mathrm{~cm}^{-2}$ are given. Taken from [70].

Additional information about the structure of the water ice mantle grown through surface reactions has also been retrieved experimentally in the case of channel (ii) (starting from $\mathrm{O}_{2}+\mathrm{H}$ ) $[143$, 144]. The absence of spectroscopic features associated with dangling bonds $\mathrm{OH}$ reported by $\mathrm{Oba}$ et al. [143] strongly supports a compact (non-porous) ice morphology. Such compact amorphous structure is confirmed by TPD experiments probing the surface of newly formed water molecules [144].

The hydrogenation of ozone interplays with both channels (i) and (ii). Experimental evidence has been made that water formation via hydrogenation of ozone is efficient and that reaction (7) proceeds without a measurable energy barrier on a non porous amorphous water ice substrate [145]. Actually, the $\mathrm{O}_{2}$ molecule released from (7) will lead to water through subsequent hydrogenation of $\mathrm{O}_{2}$, as evidenced by experiments described above, while the $\mathrm{OH}$ radical formed concomitantly can react in various ways and, especially, with $\mathrm{H}$ and $\mathrm{H}_{2}$. It is interesting to note that from the experimental studies described in this section, no information can be retrieved on the respective contribution of reactions (2) and (3) since atomic beams also include non-dissociated $\mathrm{H}_{2}$ (resp. $\mathrm{D}_{2}$ ) molecules (see table 2). Yet, reaction (3) may be of importance given the relatively low barrier for reaction of $\mathrm{OH}$ with $\mathrm{H}_{2}$ and the large number of $\mathrm{H}_{2}$ molecules on the surface both under laboratory and interstellar conditions.

An important aspect is the low level of fractionation commonly measured in water when compared to other molecules. The level of deuteration in molecules can provide strong constraints to chemical models. Low upper limits for the deuteration ratio $\mathrm{HDO} / \mathrm{H}_{2} \mathrm{O}$ in solid ices $(<1 \%)$ have been reported for intermediate and high-mass protostar environments [146, 147]. In the inner region of the envelope low-mass protostars, where ices evaporate, higher values (3\%) have been reported; but these are still significantly less than what has been found for other species such as formaldehyde and methanol $[27,148]$. Recent detection of $\mathrm{D}_{2} \mathrm{O}$ confirms low $\mathrm{D}_{2} \mathrm{O} / \mathrm{HDO} / \mathrm{H}_{2} \mathrm{O}$ ratios around low mass protostars, but the interpretations of these observations are puzzling due to the difficulty to obtain reliable water gas phase abundances [149]. Monte-Carlo simulations of water gas-grain chemistry including $\sim 150$ hydrogenation reactions suggest that low D enrichment could originate from lower abundances of HD with respect to $\mathrm{H}_{2}$ adsorbed at the surface of grains, and assuming that the main HDO production is coming through the reaction between $\mathrm{OH}+\mathrm{HD}$ (reaction (7)). This effect is in addition enhanced the $\mathrm{H}$ recycling in reaction (3) that produces water. However, the importance of the $\mathrm{OH}+\mathrm{H}_{2}$ route has not been confirmed by recent experimental study [142]. In addition, we note that these calculations do not incorporate $\mathrm{OH}+\mathrm{D}$ channels that proceed via tunneling and should show strong isotopic effect [27]. Abstraction reaction competitive to $\mathrm{H}$ addition in $\mathrm{OH}$ is also notably generally not considered, although 
this effect has been pointed out by Biener et al. [140]. Thus, we conclude that a more complete reaction network for the formation of water has to be considered to address this problem.

Several theoretical studies have focused on the processes responsible for the mantle growth at the surface of the grains. The role of direct accretion of water molecules from the gas-phase has been, in particular, subject to debate $[150,151]$. It has been suggested that direct accretion of a first $\mathrm{H}_{2} \mathrm{O}$ layer on bare grains is required to initiate the ice-mantle formation in order to circumvent the expected desorption induced by the sequence of reactions (1) and (2) that are strongly exothermic [151]. Recent calculations of $\mathrm{O}$ and $\mathrm{H}$ recombination on graphite ( $\mathrm{LH}$ mechanism) show that $\mathrm{OH}$ is produced with high level of internal energy, similarly to what has been found in case of $\mathrm{H}-\mathrm{H}$ recombination. By contrast to the $\mathrm{H}_{2}$ case however, the authors point out that desorption after formation is much less probable due to the existence of a quasi-stationary state where $\mathrm{OH}$ oscillates in a physisorption well with high degree of vibrational and rotational energy. This intermediate state favor multiple collisions with the surface and thermal equilibrium, preventing the desorption to occur [152]. The formation of water from $\mathrm{H}$ and $\mathrm{O}$-atoms has also been investigated theoretically with an embedded cluster approach on olivine (forsterite (010)). The $\mathrm{H}$-atoms are likely to be (directly or indirectly) chemisorbed. Once this first $\mathrm{H}$ atom chemisorbs, it creates a defect (localized surface electron), that directs next impinging $\mathrm{O}$ atoms to strongly chemisorb in a neighbouring site [153]. Consequently, the two atoms can form $\mathrm{OH}$ (or $\mathrm{H}_{2}$ ) and subsequent $\mathrm{H}$ adsorption produces water, with substantial exo-thermicity. In this case, the strong coupling of the chemisorbed species with the surface can quench the prompt desorption upon formation. These calculations indicate that pristine olivine surfaces should be good catalysts for water formation, with low product excitation and high reaction efficiencies.

In the ISM, UV photons can dissociate the species present on the surface. The photofragments can recombine, but a fraction of newly formed molecules are released in the gas phase. This has been shown to contribute to the total photodesorption rate of water ice for example $[154,155]$. In a very recent study based on Monte-Carlo simulations, Cazaux et al. [156] confirm that water ice that cover dust grains can only exist in shielded environment, where UV field isn't too important. This study mainly show that the chemistry on bare grains has a strong impact on gas phase chemistry due to the exo-thermicity of number of reactions occurring on the surface. As pointed out by the authors, further studies are highly needed to assess these estimates and to better understand the effect of grain surface chemistry on the gas phase composition

\section{CONCLUSIONS AND FUTURE PROSPECTS}

Much progress has been done in the last years in the characterization of heterogeneous chemical processes relevant for the ISM. In the $\mathrm{H}_{2}$ case, studies conducted at low temperature, with the aim to reproduce astrophysical conditions have been supported by models and initiated numerous studies. Detailed investigations on well-defined graphite surface have enabled more direct comparison between experiments and high level of molecular dynamics calculations (either full quantum calculations or classical trajectories). For the first time, a surface catalytic effect involving site specific chemisorbed species has been revealed and open new routes for the formation of $\mathrm{H}_{2}$ at high temperatures.

Many studies have been focused on ices, with important experimental effort to confirm formaldehyde and methanol formation routes from accretion of $\mathrm{H}$ and $\mathrm{CO}$ species. Although grain surface specific effect has been discovered in this case, prediction of deuteration fractionation after evaporation of ices remains difficult. Upcoming investigations should further characterize $\mathrm{H} / \mathrm{D}$ and $\mathrm{OH}$ formation H/D exchange at high temperatures.

The understanding of water formation through solid state reactions is still at its early stage, especially on bare grain surfaces. Recent results suggest that the processes are more complex than previously thought and further experimental efforts are required to disentangle the different pathways and derive the full reaction scheme, that in fact strongly depends on the physical conditions (gas phase environment and surface composition and temperature). It would especially be of great interest to study in detail the 
reactivity of the $\mathrm{OH}$ radical. This species indeed plays a central role in these solid state reactive processes and might be the key to achieve a better understanding of water formation in the interstellar medium. The importance of $\mathrm{OH}$ has also been evidenced for the formation of $\mathrm{CO}_{2}$ [157] (not discussed in this paper). These experiments re-opened the question of $\mathrm{CO}_{2}$ formation at very low temperature, that was thought to occur either in CO/O/ASW mixtures after thermal activation $(\mathrm{T}>140 \mathrm{~K})$ [158], or under UV [159] or charged particles irradiation [160]. Experiments conducted via co-deposition of the reactants should be further explored, since it appears that such approach enables observation of intermediate reactive species. Difficulties to understand the deuterium fractionation in water are noticeable. Observations of water in star forming regions, a key program of Herschel, should provide important new constraints to address this problem and more generally for the gas-grain chemistry.

Finally, two types of surfaces have been discussed. On bare grains surfaces (either graphite or olivine), specific chemisorbed states were found to play a real catalytic role, by affecting dramatically the barriers for reaction. By contrast, water ice does not appear to show so strong effect, since barriers are in most cases predictable based on gas phase reactions. This point should be however explored in more detail in the next future with the help of ab-initio calculations, and in light of more complete gasgrain reaction network. When the chemistry is driven by physisorbed states, the actual reactions rates are mostly dependent on the sticking probabilities, diffusion and residence times. Hence the surface topology, surface temperature and coverage, rather than the chemical nature of the substrate, are playing major roles in this case.

Other experimental approaches well-suited to study structural and optical properties of isolated nanograins have not been discussed in the present paper but can provide important details for the chemistry. This concerns for example ion-trap devices, clusters or nanograins particles of various sizes, prepared by laser pyrolysis, combustion in flames and other techniques. Taking into account the large variety of material probably present in the ISM, these approaches are necessary to complete our understanding of heterogeneous chemistry and lead to an indirect characterization of interstellar dust, through the solid state chemistry.

\section{References}

[1] A. Tielens, W. Hagen, Astron. Astrophys. 114, 245 (1982)

[2] A. Tielens, S. B. Charnley, Origins of Life and Evolution of Biospheres 27, 23 (1997)

[3] E. Herbst, Annu. Rev. Phys. Chem. 46, 27 (1995)

[4] E. Herbst, in Solid State Astrochemistry, edited by V. Pirronello, J. Krelowski, and G. Manico (Kluwer Acaddemic Publishers, 2000), Vol. 120.

[5] P. Ayotte, R. S. Smith, K. P. Stevenson, Z. Dohnalek, G. A. Kimmel, B. D. Kay, J. Geophys. Res. 106, 33387 (2001)

[6] F. Le Petit, E. Roueff, E. Herbst, Astron. Astrophys. 417, 993 (2004)

[7] T. P. Snow, A. N. Witt, Astrophys. J. 468, L65 (1996)

[8] N. Grevesse, M. Asplund, A. J. Sauval, Space Sci. Rev. 130, 105 (2007)

[9] E. Herbst, Q. Chang, H. M. Cuppen, Journal of Physics: Conference Series 6, 18 (2005)

[10] E. L. Gibb, D. C. B. Whittet, W. A. Schutte, A. C. A. Boogert, Astrophys. J. 536, 347 (2000)

[11] E. Dartois, Spa. Sci. Rev. 119, 293 (2005)

[12] C. Knez, A. C. A. Boogert, K. M. Pontoppidan, J. Kessler-Silacci, Astrophys. J. 635, L145 (2005)

[13] A. C. A. Boogert, K. M. Pontoppidan, C. Knez, F. Lahuis, Astrophys. J. 678, 985 (2008)

[14] Y. Kuan, L. E. Snyder, The astrophysical journal 470, 981 (1996)

[15] S. Cazaux, A. Tielens, C. Ceccarelli, A. Castets, Astrophys. J. 593, L51 (2003)

[16] S. Bottinelli, C. Ceccarelli, B. Lefloch, J. P. Williams, Astrophys. J. 615, 354 (2004)

[17] S. Bottinelli, C. Ceccarelli, J. P. Williams, B. Lefloch, Astron. Astrophys. 463, 601 (2007) 
[18] L. d'Hendecourt, L. J. Allamandola, R. J. A. Grim, J. M. Greenberg, Astron. Astrophys. 158, 119 (1986)

[19] L. J. Allamandola, S. A. Sandford, G. J. Valero, Icarus 76, 225 (1988)

[20] M. P. Bernstein, S. A. Sandford, L. J. Allamandola, S. Chang, M. A. Scharberg, Astrophys. J. 454, 327 (1995)

[21] M. P. Bernstein, L. J. Allamandola, S. A. Sandford, in Life Sciences: Complex Organics in Space, edited by F. Raulin and J. M. Greenberg (1997), Vol. 19, pp. 991.

[22] W. A. Schutte, in Hypervelocity Impacts in Space and Planetology, edited by J. A. M. McDonnell, T. Spohn, D. Mohlmann, and J. Klinger (1997), Vol. 20, pp. 1629.

[23] P. A. Gerakines, M. H. Moore, Icarus 154, 372 (2001)

[24] A. Crapsi, P. Caselli, C. M. Walmsley, P. C. Myers, M. Tafalla, C. W. Lee, T. L. Bourke, Astrophys. J. 619, 379 (2005)

[25] H. Roberts, E. Herbst, T. J. Millar, The astrophysical journal 591, L41 (2003)

[26] L. Colangeli, T. Henning, J. R. Brucato, D. Clement, Astron. Astrophys. Rev. 11, 97 (2003)

[27] B. Parise, PhD Thesis (2004)

[28] L. d'Hendecourt, E. Dartois, Spectrochim. Act. 57, 669 (2001)

[29] P. Ehrenfreund, H. J. Fraser, in Proceedings of the NATO Advanced Study Institute on Solid state astrochemistry., edited by J. K. a. G. M. Valerio Pirronello (Kluwer Academic Publishers, Erice, Sicily, Italy, 5-15 June 2000,, 2003), Vol. 120, pp. 317.

[30] T. Chiavassa, F. Borget, J. P. Aycard, E. Dartois, L. d'Hendecourt, Actualite Chimique, 12 (2005)

[31] V. Mennella, J. R. Brucato, L. Colangeli, P. Palumbo, A. Rotundi, in Workshop Molecules in Space and in the Laboratory, Vol 67, Conference Proceedings, edited by I. Porceddu and S. Aiello (Soc Italiana Fisica, Bologna, 2000), Vol. 67, pp. 33.

[32] E. Dartois, G. M. M. Caro, D. Deboffle, G. Montagnac, L. d'Hendecourt, Astron. Astrophys. 432, 895 (2005)

[33] T. Henning, F. Salama, Science 282, 2204 (1998)

[34] V. Pirronello, C. Liu, J. E. Roser, G. Vidali, Astronomy \& Astrophysics 344, 681 (1999)

[35] V. Pirronello, O. Biham, C. Liu, L. O. Shen, G. Vidali, Astrophysical Journal 483, L131 (1997)

[36] V. Mennella, Astrophysical Journal 647, L49 (2006)

[37] V. Mennella, Astrophys. J. Lett. 684, L25 (2008)

[38] P. Ehrenfreund, H. J. Fraser, J. Blum, J. H. E. Cartwright, Planet. Space Sci. 51, 473 (2003)

[39] Z. Dohnálek, G. A. Kimmel, P. Ayotte, R. S. Smith, B. D. Kay, J. Chem. Phys. 118, 364 (2003)

[40] E. Mayer, R. Pletzer, Nature 319, 298 (1986)

[41] G. A. Kimmel, K. P. Stevenson, Z. Dohnálek, R. S. Smith, B. D. Kay, J. Chem. Phys. 114, 5284 (2001)

[42] M. E. Palumbo, J. Phys. Conf. Ser. 6, 211 (2005)

[43] M. E. Palumbo, Astron. Astrophys. 453, 903 (2006)

[44] M. E. Palumbo, G. A. Baratta, G. Leto, G. Strazzulla, J. Mol. Struct. 972, 64 (2010)

[45] V. Pirronello, C. Liu, L. Y. Shen, G. Vidali, Astrophysical Journal 475, L69 (1997)

[46] G. Vidali, V. Pirronello, C. Liu, L. Y. Shen, Astrophysical Letters \& Communications 35, 423 (1998)

[47] N. Katz, I. Furman, O. Biham, V. Pirronello, G. Vidali, Astrophysical Journal 522, 305 (1999)

[48] J. S. Mathis, W. Rumpl, K. H. Nordsieck, Astrophys. J. 217, 425 (1977)

[49] M. P. Collings, M. A. Anderson, R. Chen, J. W. Dever, S. Viti, D. A. Williams, M. R. S. McCoustra, Mon. Not. R. Astron. Soc. 354, 1133 (2004)

[50] D. Burke, W. Brown, Phys. Chem. Chem. Phys. 12, 5947 (2010)

[51] K. Acharyya, G. W. Fuchs, H. J. Fraser, E. F. van Dishoeck, H. Linnartz, Astron. Astrophys. 466, 1005 (2007)

[52] S. E. Bisschop, H. J. Fraser, K. I. Öberg, E. F. van Dishoeck, S. Schlemmer, Astron. Astrophys. 449, 1297 (2006) 
[53] A. Al-Halabi, E. van Dishoeck, Mon. Not. R. Astron. Soc. 382, 1648 (2007)

[54] T. Zecho, A. Guttler, X. W. Sha, B. Jackson, J. Kuppers, Journal of Chemical Physics 117, 8486 (2002)

[55] S. Morisset, Y. Ferro, A. Allouche, Chemical Physics Letters 477, 225 (2009)

[56] V. Buch, Q. Zhang, Astrophys. J. 379, 647 (1991)

[57] K. Masuda, J. Takahashi, T. Mukai, Astron. Astrophys. 330, 773 (1998)

[58] A. Al-Halabi, A. W. Kleyn, E. F. van dishoeck, G. J. Kroes, The Journal of Physical Chemistry B 106, 6515 (2002)

[59] E. Matar, F. Dulieu, H. Chaabouni, J. L. Lemaire, J. Chem. Phys., submitted (2010)

[60] D. P. Ruffle, E. Herbst, Mon. Not. R. Astron. Soc. 319, 837 (2000)

[61] H. M. Cuppen, E. Herbst, Astrophys. J. 668, 294 (2007)

[62] S. Andersson, Private com. (2010)

[63] N. Watanabe, Y. Kimura, A. Kouchi, T. Chigai, T. Hama, V. Pirronello, Astrophys. J. Lett. 714, L233 (2010)

[64] E. Matar, E. Congiu, F. Dulieu, A. Momeni, J. L. Lemaire, Astron. Astrophys. 492, L17 (2008)

[65] M. J. Gillan, J. H. Harding, R. J. Tarento, Journal of Physics C-Solid State Physics 20, 2331 (1987)

[66] S. Andersson, G. Nyman, A. Arnaldsson, U. Manthe, H. Jonsson, J. Phys. Chem. A 113, 4468 (2009)

[67] S. Cazaux, A. Tielens, Astrophysical Journal 604, 222 (2004)

[68] E. Congiu, E. Matar, L. E. Kristensen, F. Dulieu, J. L. Lemaire, Monthly Notices of the Royal Astronomical Society 397, L96 (2009)

[69] T. R. Govers, L. Mattera, G. Scoles, J. Chem. Phys. 72, 5446 (1980)

[70] S. Ioppolo, H. M. Cuppen, C. Romanzin, E. F. v. Dishoeck, H. Linnartz, Astrophys. J. 686, 1474 (2008)

[71] K. I. Öberg, E. C. Fayolle, H. M. Cuppen, E. F. Van Dishoeck, H. Linnartz, Astron. Astrophys. 505, 183 (2009)

[72] R. S. Smith, C. Huang, B. D. Kay, J. Chem. Phys. B 101, 6123 (1997)

[73] R. J. Gould, E. E. Salpeter, Astrophysical Journal 138, 393 (1963)

[74] S. Cazaux, A. Tielens, Astrophysical Journal 575, L29 (2002)

[75] H. M. Cuppen, E. Herbst, Monthly Notices of the Royal Astronomical Society 361, 565 (2005)

[76] S. Morisset, F. Aguillon, M. Sizun, V. Sidis, Journal of Chemical Physics 121, 6493 (2004)

[77] S. Morisset, F. Aguillon, M. Sizun, V. Sidis, Journal of Chemical Physics 122 (2005)

[78] D. Bachellerie, M. Sizun, F. Aguillon, V. Sidis, Journal of Physical Chemistry A 113, 108 (2009)

[79] J. S. A. Perry, S. D. Price, Astrophysics and Space Science 285, 769 (2003)

[80] S. C. Creighan, J. S. A. Perry, S. D. Price, Journal of Chemical Physics 124 (2006)

[81] F. Islam, E. R. Latimer, S. D. Price, Journal of Chemical Physics 127 (2007)

[82] E. R. Latimer, F. Islam, S. D. Price, Chemical Physics Letters 455, 174 (2008)

[83] L. Amiaud, F. Dulieu, J. H. Fillion, A. Momeni, J. L. Lemaire, Journal of Chemical Physics 127 (2007)

[84] S. Casolo, O. M. Lovvik, R. Martinazzo, G. F. Tantardini, Journal of Chemical Physics 130 (2009)

[85] E. Habart, F. Boulanger, L. Verstraete, C. M. Walmsley, G. P. des Forets, Astronomy \& Astrophysics 414, 531 (2004)

[86] T. Zecho, A. Guttler, X. W. Sha, D. Lemoine, B. Jackson, J. Kuppers, Chemical Physics Letters 366, 188 (2002)

[87] L. Jeloaica, V. Sidis, Chemical Physics Letters 300, 157 (1999)

[88] X. W. Sha, B. Jackson, D. Lemoine, Journal of Chemical Physics 116, 7158 (2002)

[89] X. W. Sha, B. Jackson, D. Lemoine, B. Lepetit, Journal of Chemical Physics 122 (2005)

[90] Y. Ferro, F. Marinelli, A. Jelea, A. Allouche, Journal of Chemical Physics 120, 11882 (2004) 
[91] N. Rougeau, D. Teillet-Billy, V. Sidis, Chemical Physics Letters 431, 135 (2006)

[92] L. Hornekaer, E. Rauls, W. Xu, Z. Sljivancanin, Physical Review Letters 97 (2006)

[93] L. Hornekaer, Z. Sljivancanin, W. Xu, R. Otero, Physical Review Letters 96 (2006)

[94] Y. Ferro, D. Teillet-Billy, N. Rougeau, V. Sidis, S. Morisset, A. Allouche, Physical Review B 78 (2008)

[95] D. Bachellerie, M. Sizun, D. Teillet-Billy, N. Rougeau, V. Sidis, Chemical Physics Letters 448, 223 (2007)

[96] S. Cazaux, M. Spaans, Astronomy \& Astrophysics 496, 365 (2009)

[97] H. M. Cuppen, L. Hornekaer, Journal of Chemical Physics 128 (2008)

[98] L. Hornekaer, W. Xu, R. Otero, E. Laegsgaard, F. Besenbacher, Chemical Physics Letters 446, 237 (2007)

[99] Y. Ferro, S. Morisset, A. Allouche, Chemical Physics Letters 478, 42 (2009)

[100] K. Hiraoka, T. Miyagoshi, T. Takayama, K. Yamamoto, Y. Kihara, Astrophys. J. 498, 710 (1998)

[101] K. Hiraoka, T. Sato, S. Sato, N. Sogoshi, T. Yokyoma, H. Takashima, S. Kitagawa, Astrophys. J. 577, 265 (2002)

[102] N. Watanabe, A. Kouchi, Astrophys. J. 571, L173 (2002)

[103] H. Hidaka, N. Watanabe, T. Shiraki, A. Nagaoka, A. Kouchi, Astrophys. J. 614, 1124 (2004)

[104] N. Watanabe, A. Nagaoka, T. Shiraki, A. Kouchi, Astrophys. J. 616, 638 (2004)

[105] N. Watanabe, A. Nagaoka, H. Hidaka, T. Shiraki, T. Chigai, A. Kouchi, Planet. Spac. Sci. 54, 1107 (2006)

[106] H. Hidaka, A. Kouchi, N. Watanabe, Journal of Chemical Physics 126 (2007)

[107] N. Watanabe, O. Mouri, A. Nagaoka, T. Chigai, A. Kouchi, V. Pirronello, Astrophys. J. 668, 1001 (2007)

[108] H. Hidaka, N. Miyauchi, A. Kouchi, N. Watanabe, Chem. Phys. Lett. 456, 36 (2008)

[109] G. W. Fuchs, H. M. Cuppen, S. Ioppolo, C. Romanzin, Astron. Astrophys. 505, 629 (2009)

[110] N. Watanabe, A. Kouchi, Prog. Surf. Sci. 83, 439 (2008)

[111] N. Watanabe, T. Shiraki, A. Kouchi, Astrophys. J. 588, L121 (2003)

[112] R. A. Zhitnikov, Y. U. Dmitriev, Astron. Astrophys. 386, 1129 (2002)

[113] C. Pirim, L. Krim, C. Laffon, P. Parent, F. Pauzat, J. Pilmé, Y. Ellinger, J. Phys. Chem. A 114, 3320 (2010)

[114] D. E. Woon, Astrophys. J. 569, 541 (2002)

[115] S. E. Bisschop, G. W. Fuchs, E. F. van Dishoeck, H. Linnartz, Astron. Astrophys. 474, 1061 (2007)

[116] G. W. Fuchs, K. Acharyya, S. E. Bisschop, K. I. Oberg, Farad. Discuss. 133, 331 (2006)

[117] N. Miyauchi, H. Hidaka, T. Chigai, A. Nagaoka, N. Watanabe, A. Kouchi, Chem. Phys. Lett. 456, 27 (2008)

[118] A. Nagaoka, N. Watanabe, A. Kouchi, J. Phys. Chem. A 111, 3016 (2007)

[119] A. Nagaoka, N. Watanabe, A. Kouchi, Astrophys. J. 624, L29 (2005)

[120] M. Watanabe, H. Hidaka, A. Nagaoka, A. Kouchi, in Molecules in Space \& Laboratory, edited by J. L. L. a. F. Combes (Paris, 2007).

[121] B. Parise, C. Ceccarelli, A. Tielens, E. Herbst, Astron. Astrophys. 393, L49 (2002)

[122] B. Parise, C. Ceccarelli, A. Tielens, E. Herbst, in Chemistry as a Diagnostic of Star Formation, edited by M. Fich (2003), pp. 378.

[123] B. Parise, C. Ceccarelli, A. Tielens, A. Castets, E. Caux, B. Lefloch, S. Maret, Astron. Astrophys. 453, 949 (2006)

[124] A. Ratajczak, E. Quirico, A. Faure, B. Schmitt, C. Ceccarelli, Astron. Astrophys. 496, L21 (2009)

[125] S.-C. Park, K.-H. Jung, H. Kang, J. Chem. Phys. 121, 2765 (2004)

[126] C.-W. Lee, P.-R. Lee, Y.-K. Kim, H. Kang, J. Chem. Phys. 127, 1 (2007)

[127] D. E. Brown, A. S. Bolina, Mon. Not. R. Astron. Soc. 374, 1006 (2007) 
[128] N. J. Sack, R. A. Baragiola, Phys. Rev. B 48, 9973 (1993)

[129] J. V. Keane, A. G. G. M. Tielens, A. C. A. Boogert, W. A. Schutte, D. C. B. Whittet, Astron. Astrophys. 376, 254 (2001)

[130] E. L. Gibb, D. C. B. Whittet, A. C. A. Boogert, A. G. G. M. Tielens, ApJ Suppl.151, 35 (2004)

[131] A. G. G. M. Tielens, L. J. Allamandola, J. Bregman, J. Goebel, L. D’Hendecourt, F. C. Witteborn, Astrophys. J. 287, 697 (1984)

[132] L. B. D’Hendecourt, L. J. Allamandola, J. M. Greenberg, Astron. Astrophys. 152, 130 (1985)

[133] T. I. Hasegawa, E. Herbst, C. M. Leung, Astrophysical Journal Supplement Series 82, 167 (1992)

[134] M. J. Loeffler, B. D. Teolis, R. A. Baragiola, J. Chem. Phys. 124, 10472 (2006)

[135] P. A. Gerakines, W. A. Schutte, P. Ehrenfreund, Astron. Astrophys. 312, 289 (1996)

[136] C. J. Bennett, R. I. Kaiser, Astrophys. J. 635, 1362 (2005)

[137] P. D. Cooper, M. H. Moore, R. L. Hudson, The Journal of Physical Chemistry A 110, 7985 (2006)

[138] R. Klein, M. D. Scheer, Journal of Chemical Physics 31, 278 (1959)

[139] P. A. Giguère, D. Chin, J. Chem. Phys. 31, 1685 (1959)

[140] J. Biener, E. Lang, C. Lutterloh, K. J., J. Chem. Phys. 116, 3063 (2002)

[141] F. Dulieu, L. Amiaud, E. Congiu, J.-H. Fillion, Astron. Astrophys. 512, A30 (2010)

[142] H. M. Cuppen, S. Ioppolo, C. Romanzin, H. Linnartz, Physical Chemistry Chemical Physics (submitted)

[143] Y. Oba, N. Miyauchi, H. Hidaka, T. Chigai, N. Watanabe, A. Kouchi, Astrophys. J. 701, 464 (2009)

[144] M. Accolla, F. e.a. Dulieu, in preparation

[145] H. Mokrane, H. Chaabouni, M. Accolla, E. Congiu, F. Dulieu, M. Chehrouri, J. L. Lemaire, Astrophys. J. Lett. 705, L195 (2009)

[146] E. Dartois, W.-F. Thi, T. R. Geballe, D. Deboffle, L. d'Hendecourt, E. van Dishoeck, Astron. Astrophys. 399, 1009 (2003)

[147] B. Parise, T. Simon, E. Caux, E. Dartois, C. Ceccarelli, J. Rayner, A. Tielens, Astron. Astrophys. 410, 897 (2003)

[148] B. Parise, E. Caux, A. Castets, C. Ceccarelli, Astron. Astrophys. 431, 547 (2005)

[149] H. M. Butner, S. B. Charnley, C. Ceccarelli, S. D. Rodgers, Astrophys. J. 659, L137 (2007)

[150] A. P. Jones, D. A. Williams, Mon. Not. R. Astron. Soc. 209, 955 (1984)

[151] R. Papoular, Mon. Not. R. Astron. Soc. 362, 489 (2005)

[152] H. Bergeron, N. Rougeau, V. Sidis, M. Sizun, D. Teillet-Billy, F. Aguillon, Journal of Physical Chemistry A 112, 11921 (2008)

[153] T. P. M. Goumans, C. R. A. Catlow, W. A. Brown, J. Kästner, P. Sherwood, Phys. Chem. Chem. Phys. 11, 5432 (2009)

[154] K. Öberg, H. Linnartz, R. Visser, E. van Dishoeck, Astrophys. J. 693, 1209 (2009)

[155] S. Andersson, A. Al-Halabi, G. J. Kroes, E. F. van Dishoeck, J. Chem. Phys. 124 (2006)

[156] S. Cazaux, V. Cobut, M. Marseille, M. Spaans, P. Caselli, Astron. Astrophys., submitted (2010)

[157] Y. Oba, N. Watanabe, A. Kouchi, T. Hama, V. Pirronello, Astrophys. J. Lett. 712, L174 (2010)

[158] J. Roser, G. Vidali, G. Manicò, V. Pirronello, Astrophys. J. 555, L61 (2001)

[159] M. Watanabe, A. Kouchi, Astrophys. J. 567, 651 (2002)

[160] M. H. Moore, R. Khanna, B. Donn, J. Geophys. Res. 96, 17541 (1991) 